\title{
MONTE CARLO SIMULATIONS OF THE ASHKIN-TELLER MODEL
}

\author{
L. DĘBSKI \\ Institute of Physics, Adam Mickiewicz University \\ Umultowska 85, 61-614 Poznań, Poland
}

\begin{abstract}
Monte Carlo simulations are used to analyse the behaviour of the extended Ashkin-Teller model in two (2D) and three (3D) dimensions from the ratio of the square of the second moment of the order parameter to its fourth moment. The critical surface in three dimensional parameter space is calculated in regions where the Ising transitions are expected both for the ferro-and antiferromagnetic regions.
\end{abstract}

PACS numbers: 75.10.Hk, 75.40.Mg, 64.70.-p

\section{Introduction}

The Ashkin-Teller (A-T) model is a simple extension of the Ising model but its critical properties reveal new interesting features [1]. It can be viewed as a combination of two Ising models with some four-order interaction. If the third-order terms are included [2,3], the extended A-T model is described by the Hamiltonian analysis

$$
-\beta \mathcal{H}=H=\sum_{i, j}\left[K_{2}\left(s_{i} s_{j}+\sigma_{i} \sigma_{j}\right)+K_{4} s_{i} s_{j} \sigma_{i} \sigma_{j}+K\left(s_{i}+s_{j}\right)\left(\sigma_{i} \sigma_{j}+1\right)\right],
$$

where $\beta=\left(k_{\mathrm{B}} T\right)^{-1}$, the summation is taken over nearest neighbour sites of a square or cubic lattice and spins $s, \sigma= \pm 1$ are the Ising variables. The temperature is included in the interaction parameters of the model, so that the two-spin interaction parameter $K_{2}$, the four-spin interaction parameter $K_{4}$, and the odd interaction parameter $K$ are dimensionless. The standard A-T model corresponds to $K=0$.

The properties of the extended A-T model were investigated within the molecular-field approximation (MFA) [2] as well as the molecular field renormalization group (MFRG) method [3]. The standard A-T model in 2 and 3 dimensions was examined $[4,5]$ within the Monte Carlo methods and the series analysis, yielding the approximate phase diagrams.

Here, we verify the Ising-like transitions in the extended model (1) in 2D, using the Monte Carlo simulations. We also address the problem of Ising transitions for the standard model in 3D. The latter seems to exhibit the tricritical point in the antiferromagnetic region [4] but both the simulations and the series analysis supporting this picture, are too short to give a definite answer. 


\section{Description of the method}

In our investigation of the model (1) we generated (by computer) the equilibrium configurations of the finite size samples of spins for fixed values of the model parameters. Periodic boundary conditions were imposed and thermalization of the initial configurations was applied.

Gibbs distribution was sampled using the Metropolis algorithm [6]. We started with some initial configuration $\alpha$ of spins and a new configuration $\alpha^{\prime}$ of the system was generated from $\alpha$ by the repetive application of the important sampling procedure when flipping successive spins on the lattice.

The spins may be selected at random, or each of the spins in the sample may be reversed in turn (which is the case here). We reverse the spin or do not do it according to some initially chosen transition probability and when each spin of the sample has been visited once (on the average or consecutively) we carried out one Monte Carlo step (MCS) per spin. Either of these procedures ensures that the accessibility criterion, which states that it must be possible to evolve the system from a given starting point to any of its other configurations by applying the evolution rule a sufficiently large number of times, is satisfied.

In order to decide whether to accept a single spin-flip or not, we compared the energies of the new and old configurations. If the energy change $E_{\alpha^{\prime}}-E_{\alpha}$ was negative, then the new configuration was automatically accepted; if, however, it was positive, the new configuration was accepted with a probability $\exp \left[-\beta\left(E_{\alpha^{\prime}}-E_{\alpha}\right)\right]$. Physically, it means that both configurations are in equilibrium and none of them arises at the expense of the other. Using this method, we generated configurations which allowed us to calculate physical quantities in a direct way.

The transition point was determined $[7,8]$ from the analysis of the fourth order cumulant

$$
Q_{L}=\frac{\left\langle M^{2}\right\rangle_{L}^{2}}{\left\langle M^{4}\right\rangle_{L}}
$$

where $\left\langle M^{n}\right\rangle$ denotes the $n$-th power of an order parameter averaged over the assembly of independent samples of the size $L \times L$ or $L \times L \times L$ in 2D and 3D, respectively. For $T>T_{\mathrm{c}}$ and $L \gg \xi$, where $\xi$ denotes the correlation length, $Q_{L}$ tends towards $1 / 3$ which corresponds to a Gaussian distribution. For $T<T_{\mathrm{c}}$ and $L \gg \xi, Q_{L}$ tends to 1 . For $L \ll \xi, Q_{L}$ varies only weakly with temperature and linear dimension, and stays very close to the value of 0.856 [8] in two dimensions and 0.628 [9] in three dimensions - the values achieved at the critical point. This number is characteristic of the corresponding Ising universality class. This behaviour of the cumulant is useful for determination of $T_{c}$. One may plot $Q_{L}$ versus $T$ for various $L$ and estimate $T_{\mathrm{c}}$ from the intersection point of these curves [7].

\section{Results}

We performed our simulations on the two-dimensional square lattice and for the regions of the parameter space $\left(K_{2}, K_{4}, K\right)$, where the phase transitions are expected to be of the Ising type. It is known [1] that the line going from the Potts point $\left(K_{2}=K_{4}=0.275\right)$ to the Ising point $\left(K_{2}=0, K_{4}=0.441\right)$ is of the Ising 
type. We expected that its continuation towards nonzero $K$ values would exhibit the same behaviour. Also a line which begins at the point $\left(K_{2}=0, K_{4}=-0.441\right)$ has the Ising-like critical exponents and therefore, we performed calculations of the surface which is a continuation of this line for nonzero $K$.

We used $\langle s \sigma\rangle$ as an order parameter $M$ because $s$ is never critical for $K \neq 0$ and $\langle\sigma\rangle$ takes small values even in temperatures far from the critical one. Figure 1 shows a three-dimensional phase diagram in the $\left(K_{2}, K_{4}, K\right)$ space of parameters for the model (1) on the square lattice.

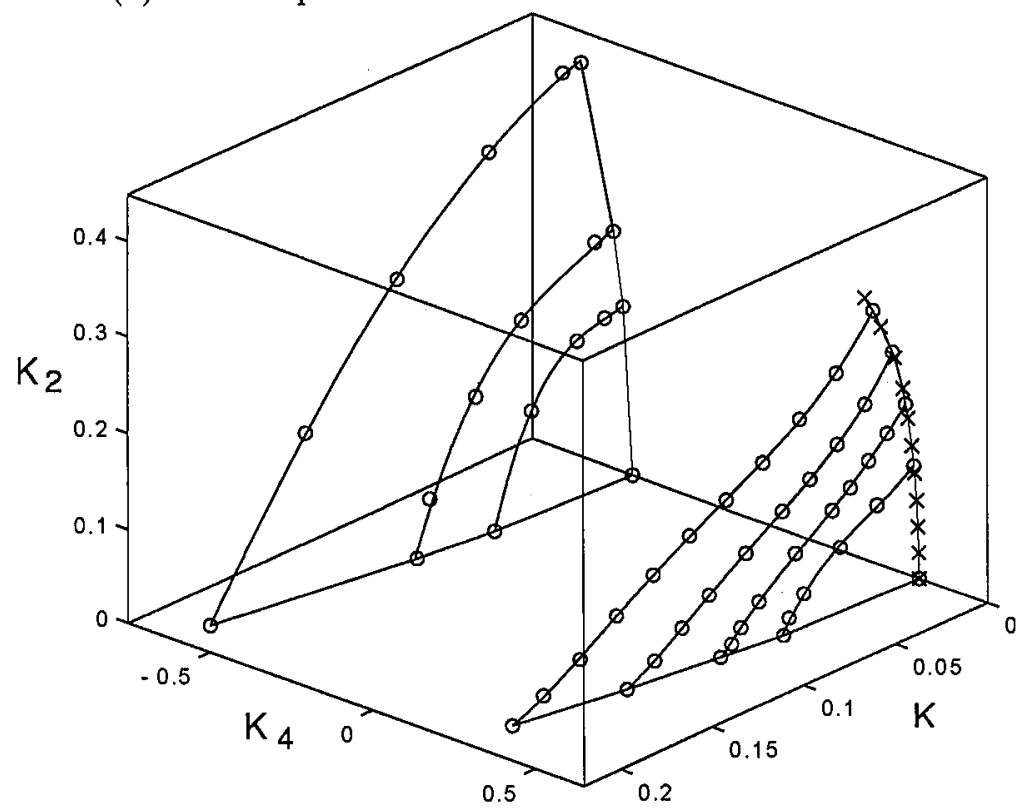

Fig. 1. The critical surface in the space of parameters for the Ashkin-Teller model in 2D. Circles denote the measured values, stars denote the values from the transfer matrix technique. Lines are drawn to guide eyes.

In three dimensions and for $K=0$, we investigated the ferromagnetic and antiferromagnetic regions of the parameters $\left(K_{2}, K_{4}\right)$ with Ising-type phase transitions, using systems with linear size $L \leq 25$. The corresponding estimates of the critical couplings $K_{2}^{\mathrm{c}}$ as a function of $K_{4}^{\mathrm{c}}$ are presented in Table. Our results for $K_{2}^{\mathrm{c}}$, denoted as $K_{2}^{\mathrm{f}}, K_{2}^{\mathrm{af}}$ (for the ferro- and antiferromagnetic region, respectively), are given in the second and the fifth column whereas previous estimates $K_{2}^{\mathrm{DB}}$ are displayed in the third and the sixth column.

In the Ising limit $K_{2}=0$ we estimated the critical coupling at $K_{4}^{c}=0.2215(4)$ which agrees within the error bars with that found by Talapov et al. [9], using the cluster processor and a newly built special-purpose computer.

\section{Discussion}

Our main result reported here is the three-dimensional phase diagram in $2 \mathrm{D}$ shown in Fig. 1 and the Monte Carlo data for the critical couplings in 3D given in Table. 
TABLE

The ferromagnetic and antiferromagnetic critical couplings for the Ising-type transitions in the $3 \mathrm{D}$ A-T model.

\begin{tabular}{l|l|l|c|c|l}
\hline \hline$K_{4}^{\mathrm{c}}$ & \multicolumn{1}{|c|}{$K_{2}^{\mathrm{f}}$} & $K_{2}^{\mathrm{DB}}$ & $K_{4}^{\mathrm{c}}$ & \multicolumn{1}{c|}{$K_{2}^{\mathrm{af}}$} & $K_{2}^{r \mathrm{DB}}$ \\
\hline 0.22 & 0.04 & 0.05 & -0.23 & 0.09 & 0.05 \\
0.217 & 0.066 & 0.06 & -0.242 & 0.1413 & 0.12 \\
0.213 & 0.087 & 0.078 & -0.266 & 0.208 & 0.21 \\
0.21 & 0.0996 & 0.1 & -0.36 & 0.359 & 0.36 \\
0.203 & 0.118 & 0.11 & & & \\
0.195 & 0.133 & 0.13 & & &
\end{tabular}

In 2D no evidence for the first order phase transition was found (see Ref. [6] for more details) although some MFA or MFRG results [2,3] suggested that it may occur. In 3D our results are more accurate than those of Ditzian et al. [4] and suggest that the region of the Ising line transitions is other in the antiferromagnetic part of the $K_{2}-K_{4}$ phase diagram, which may be extended with respect to that found previously [1]. As to the tricritical point in $3 \mathrm{D}$, its existence still needs more considerations. The simulations in the vicinity of non-Ising universality class have to be performed extremely carefully. The estimates given in Table are calculated from the corresponding 6 partial averages, each consisting of several millions of MCS per spin.

\section{Acknowledgments}

I would like to thank Prof. G. Kamieniarz for helpful discussions and the Committee for Scientific Research for partial support via grant No. 2 PO3B 07516. The Supercomputing and Networking Center in Poznan is also acknowledged for access to the computational facilities.

\section{References}

[1] R.J. Baxter, Exactly Solvable Models in Statistical Mechanics, Academic Press, London 1982.

[2] P. Pawlicki, J. Rogiers, Physica A 214, 277 (1995).

[3] P. Pawlicki, G. Musial, G. Kamieniarz, J. Rogiers, Physica A 242, 281 (1997).

[4] R.V. Ditzian, J.R. Banavar, G.S. Grest, L.P. Kadanoff, Phys. Rev. B 22, 2542 (1980).

[5] P. Arnold, Y. Zhang, Nuclear Phys. B 501, 473 (1997).

[6] N. Metropolis, A. Rosenbluth, M. Rosenbluth, A. Teller, E. Teller, J. Chem. Phys. 21, 1087 (1953).

[7] P. Pawlicki, G. Kamieniarz, L. Dębski, Physica A 242, 290 (1997).

[8] G. Kamieniarz, H.W.J. Blöte, J. Phys. A, Math. Gen. 26, 201 (1993).

[9] A.L. Talapov, H.W.J. Blöte, J. Phys. A, Math. Gen. 29, 5727 (1996). 